\title{
Percutaneous nephrolithotomy in patients with solitary kidney: a critical outcome analysis
}

\author{
Fabio C. M. Torricelli ${ }^{1}$, Guilherme P. Padovani ${ }^{1}$, Giovanni S. Marchini ${ }^{1}$, Fabio C. Vicentini ${ }^{1}$, Alexandre \\ Danilovic ${ }^{1}$, Sabrina T. Reis ${ }^{1}$, Miguel Srougi ${ }^{1}$, Eduardo Mazzucchi ${ }^{1}$ \\ ${ }^{1}$ Divisão de Urologia do Hospital das Clínicas da Universidade de São Paulo, Faculdade de Medicina de \\ São Paulo, Brasil
}

\section{ABSTRACT}

Purpose: To describe our experience with percutaneous nephrolithotomy (PCNL) in patients with solitary kidneys and analyze factors that can impact on intra-operative bleeding and postoperative complications.

Materials and Methods: We reviewed our stone database searching for patients with solitary kidney who underwent PCNL from Jan-05 through 0ct-13. Demographic data, stone characteristics, and intra- and postoperative outcomes were recorded. Spearman correlation was performed to assess which variables could impact on bleeding and surgical complications. Linear and logistic regressions were also performed.

Results: Twenty-seven patients were enrolled in this study. The mean age and BMI were 45.6 years and $28.8 \mathrm{Kg} / \mathrm{m}^{2}$, respectively; $45 \%$ of cases were classified as Guys 3 (partial staghorn or multiple stones) or 4 (complete staghorn) - complex cases. Stone-free rate was 67\%. Eight (29.6\%) patients had postoperative complications (five of them were Clavien 2 and three were Clavien 3). On univariate analysis only number of tracts was associated with increased bleeding $(\mathrm{p}=0.033)$ and only operative time was associated with a higher complication rate $(\mathrm{p}=0.044)$. Linear regression confirmed number of access tracts as significantly related to bleeding $(6.3,95 \% \mathrm{CI} 2.2-10.4$; $\mathrm{p}=0.005$ ), whereas logistic regression showed no correlation between variables in study and complications.

Conclusions: PCNL in solitary kidneys provides a good stone-free rate with a low rate of significant complications. Multiple access tracts are associated with increased bleeding.

\section{ARTICLE INFO}

Key words:

Hemorrhage; Kidney; Lithotripsy; Nephrostomy, Percutaneous;

Urinary Calculi

Int Braz J Urol. 2015; 41: 496-502

Submitted for publication:

July 10, 2014

Accepted after revision:

October 23, 2014

\section{INTRODUCTION}

Nephrolithiasis prevalence has been increasing (1) and patients with solitary kidneys are also at a higher risk of developing kidney stones. These patients deserve appropriate metabolic evaluation and efficient stone clearance as this condition might ultimately worsen their renal function (2).

The management of urolithiasis in patients with a solitary kidney remains a challenging scenario. Shock wave lithotripsy (SWL) and retrograde intrarenal surgery (RIRS) have similar ou- tcomes in patients with one or two functional kidneys $(3,4)$. Conversely, there is literature evidence that percutaneous nephrolithotomy (PCNL) in patients with solitary kidneys might have a higher morbidity rate when compared to patients with two functioning kidneys (5). Even though PCNL is related to potential surgical complications, e.g. bleeding, infection, lung collapse, and urinary fistula, it remains as the gold standard treatment for complex kidney stones even for patients with solitary kidneys, providing reasonable stone-free rates while preserving renal function (6-8). 
The aim of this study is to describe the experience of a large volume referral center for kidney stones management with PCNL in patients with solitary kidneys. We also performed a critical analysis of factors that can impact on intra-operative bleeding and postoperative complications.

\section{MATERIAL AND METHODS}

\section{Study Design}

After Institutional Board Review approval, we reviewed our stone database searching for patients with solitary kidneys who underwent PCNL from January 2005 through October 2013. Patients were considered to have a solitary kidney in case of congenital abnormality, contralateral nephrectomy, or solitary functioning kidney with contralateral atrophy (relative function $<5 \%$ ). Patients under 18 year-old were excluded from this study.

Pre-operative data recorded included age, gender, body mass index (BMI), American Society of Anesthesiology (ASA) classification, serum creatinine, renal function at the time of PCNL calculated by Modification of Diet in Renal Disease (MDRD) equation (estimated glomerular filtration rate - eGFR), cause of solitary kidney, laterality, renal cortex thickness measurement with ultrasound, stone burden, and Guys score (9). Intra and post-operative data comprised patient's PCNL position (prone vs. supine), number of urinary tracts, drop in serum hemoglobin and hematocrit levels (bleeding), transfusion rate, operative time (defined from the beginning of the cystoscopy until the end of nephrostomy tube placement), variation of creatinine clearance, complications according to the Clavien classification, length of hospital stay, and stone-free rate.

\section{Surgical Technique And Postoperative Image Control \\ All patients received prophylactic third- -generation cephalosporin preoperatively during anesthesia induction, or therapeutic culture-gui- ded antibiotic initiated seven days before surgery. Patients with staghorn calculi started oral antibio- tics 7 days before surgery irrespective of urinary culture status.}

Briefly, all patients were submitted to cystoscopy under general anesthesia for a 5 Fr ureteral catheter placement and retrograde pyelography. Thereafter, patients were positioned in prone or supine position according to the surgeon's preference. Upper tract access was planned by examining preoperative noncontrast computed tomography (NCCT) and intraoperative fluoroscopy. The collecting system was punctured, a guidewire was inserted, and then the urinary tract was dilated with serial dilators. After nephroscopic inspection, lithotripsy was done with ultrasonic lithotripter (Swiss Lithosclast ${ }^{\circledR}$ Master, Electro Medical System) allowing concomitant stone fragmentation and suction. Larger stone fragments were retrieved with graspers. At the end of all procedures, flexible nephroscopy was performed. An eighteen Fr nephrostomy tube was routinely left in place at least for two days. Ureteral catheter or a double $\mathrm{J}$ stent were also routinely left.

In the first postoperative day, all patients underwent NCCT and laboratory exams following our Institutional protocol. Patients with residual stones were submitted to auxiliary procedures according to the residual stone burden and location. Patients who underwent auxiliary procedures were submitted to a consented additional NCCT.

\section{Statistical analysis}

Results were expressed in proportion, mean, and standard deviation. Spearman correlation was performed to assess which variables could impact on bleeding (drop in hematocrit level) and surgical complications. Linear and logistic regression including BMI, ASA classification, baseline clearance creatinine, patient's PCNL position, number of urinary tracts, stone burden, Guys score, renal cortex thickness and operative time were performed to evaluate which variables were significantly related to bleeding and complications, respectively. Statistical analysis was performed with SPSS version 20.0 (SPSS Inc., Chicago, IL) and significance level was set up at $\mathrm{p}<0.05$.

\section{RESULTS}

Twenty-seven patients (29.6\% male) with solitary kidneys who underwent PCNL were enrol- 
led in this study. The mean ( \pm standard deviation) age and BMI were $45.6 \pm 14.6$ years and $28.8 \pm 4.7$ $\mathrm{Kg} / \mathrm{m}^{2}$, respectively. Mean stone burden was $503 \pm 222 \mathrm{~mm}^{2}$ and almost half of patients (45\%) were classified as Guys 3 (partial staghorn or multiple stones) or 4 (complete staghorn) - complex cases. Most patients (78\%) had a solitary kidney due to a stone-related event. Demographic and preoperative data are summarized in Table-1.

The majority of patients were positioned in prone position (63\%) for PCNL and had a single puncture performed (78\%). Stone-free rate after auxiliary procedures was $67 \%$. Mean drop in the hematocrit level was $8.1 \%$ and it was used to estimate the intra-operative bleeding. Blood transfusion was required in five $(18.5 \%)$ cases. Mean operative time was $138.3 \pm 36.7$ minutes and mean length of hospital stay was $5.6 \pm 3.9$ (range 2 to 16 ) days. Most patients (55.5\%) stayed in the hospital for no more than 4 days; only four patients had a hospitalization time longer than one week due to surgical complications. Eight (29.6\%) patients had postoperative complications; five of them were Clavien 2 (one urinary tract infection and four blood transfusions) and three were Clavien 3 (two urinary fistulas treated with $2 \mathrm{~J}$ placement one patient also needed a blood transfusion - and one $2 \mathrm{~J}$ displacement that required anesthesia for surgical removal). The variation between pre- and postoperative creatinine clearance was minimal. Table-2 shows intra- and postoperative data.

On univariate analysis, only the number of urinary tracts was associated with increased bleeding $(p=0.033)$ - Figure-1 - and only operative time was associated with a higher complication rate $(p=0.044)$ - Table-3. Linear regression con-

Table1 - Demographic and preoperative data.

\begin{tabular}{lc}
\hline Demographic data & \\
\hline Age (years) & $25.6 \pm 14.6$ \\
Gender (Male) & $28.6 \% 4.7$ \\
BMI (Kg/m²) & $37 \%$ ASA $1 / 48 \%$ ASA 2 / $15 \%$ ASA 3 \\
ASA score & $1.5 \pm 0.8$ \\
Serum creatinine $(\mathrm{mg} / \mathrm{dL})$ & $60.5 \pm 32.0$ \\
Creatinine Clearence $\left(\mathrm{mL} / \mathrm{min} / 1.73 \mathrm{~m}^{2}\right)$ & \\
Preoperative data & $48 \%$ \\
Laterality (Right) & $1.9 \pm 0.6$ \\
Renal Cortex Thickness (cm) & $503 \pm 222$ \\
Stone Burden (mm 2$)$ & $11 \%$ Guys $1 / 33 \%$ Guys $2 / 41 \%$ Guys $3 / 4 \%$ Guys 4 \\
Guys score & \\
Solitary kidney cause & $21(78 \%)$ \\
Lithiasis & $3(10 \%)$ \\
Renal agenesia & $1(4 \%)$ \\
Kidney cancer & $1(4 \%)$ \\
Abdominal trauma & $1(4 \%)$ \\
Kidney donation &
\end{tabular}

$\mathbf{B M I}=$ Body mass index; $\mathbf{A S A}=$ American Society of Anesthesiology 
Table 2 - Intra- and postoperative data.

\begin{tabular}{lc}
\hline PCNL Position (Prone / Supine) & $63 \% / 37 \%$ \\
\hline Number of Urinary Tracts & $78 \%$ One / 22\% Two \\
Hb level drop (mg/dL) & 2.7 (Initial 13.4 - Final 10.7) \\
Ht level drop (\%) & 8.1 (Initial 40.7 - Final 32.6) \\
Transfusion ( $\mathrm{n} ; \%)$ & 5 cases; 18.5 \\
Operative time (min) & $138.3 \pm 36.7$ \\
Length of hospital stay (days) & $5.6 \pm 3.9$ \\
Complications & $8(29.6 \%)$-Clavien 2: $62.5 \%$ Clavien 3: $37.5 \%$ \\
Creatinine variation (mg/dL) & 0.1 (Initial 1.5-Final 1.4) \\
Creatinine Clearence variation (mL/min/1.73m²) & 0.5 (Initial 60.5-Final 60) \\
Stone-free rate (\%) & 67 \\
\hline
\end{tabular}

$\mathbf{H b}=$ Hemoglobin; $\mathbf{H t}=$ Hematocrit

Figure 1 - Correlation between number of urinary tracts and drop in hematocrit level.

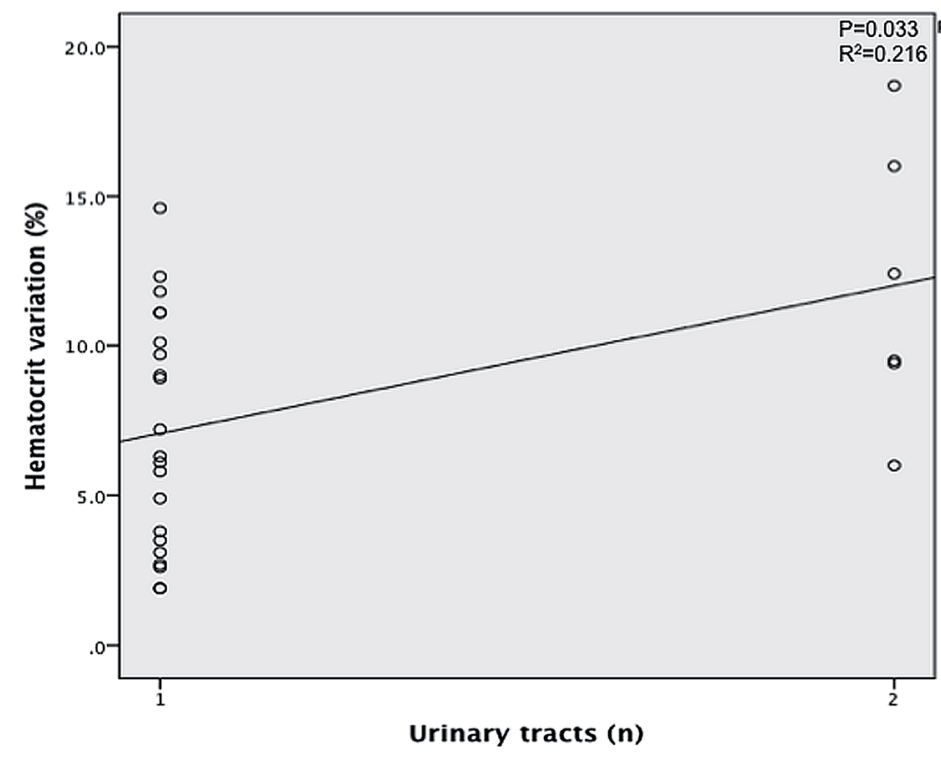

firmed number of urinary tracts as significantly related to bleeding - drop in the hematocrit level (6.3, 95\%CI 2.2-10.4; $\mathrm{p}=0.005$ ), whereas logistic regression showed no correlation between variables in study and complications.

\section{DISCUSSION}

We studied patients with solitary kidneys who underwent PCNL in a referral center for management of kidney stones to compare our ou- 
Table 3 - Univariate analysis - Sperman correlation.

\begin{tabular}{lcccc}
\hline & \multicolumn{2}{c}{ Bleeding } & \multicolumn{2}{c}{ Complications } \\
\hline BMI & $\mathrm{p}$-value & $\mathrm{R}^{2}$ linear & $\mathrm{p}$-value & $\mathrm{R}^{2}$ linear \\
ASA classification & 0.170 & 0.085 & 0.361 & 0.038 \\
Initial serum creatinine & 0.793 & 0.003 & 0.910 & $<0.001$ \\
Initial clearence of creatinine & 0.512 & $<0.001$ & 0.697 & 0.052 \\
Patient's position & 0.709 & 0.024 & 0.938 & 0.004 \\
Number of urinary tracts & 0.508 & 0.015 & 0.420 & 0.026 \\
Stone burden & 0.033 & 0.216 & 0.450 & 0.023 \\
Guys score & 0.971 & 0.002 & 0.918 & 0.003 \\
Renal cortex tickness & 0.653 & 0.017 & 0.978 & $<0.001$ \\
Operative time & 0.631 & 0.010 & 0.735 & 0.008 \\
\hline
\end{tabular}

$\mathbf{B M I}=$ Body mass index; $\mathbf{A S A}=$ American Society of Anesthesiology

tcomes with others centers worldwide; then we analyzed factors that could impact on intraoperative bleeding and postoperative complications. Compared to the largest series of PCNL in solitary kidneys reported in the literature (Clinical Research Office of the Endourological Society - CROES) (5), both studies showed reasonable stone-free rates $(>65 \%)$ with high proportion of transfusion and minor complication rates. In our cases, almost 25\% of our PCNLs were done with two percutaneous accesses, which was significantly related to a higher bleeding rate, compared to only $10.6 \%$ of cases from CROES study. We also had a high number of complex cases (Guys 3 and 4) that can have impacted on our outcomes, although it was not significantly associated with our analyzed results. Eight patients had postoperative complications, however only $3(11.1 \%)$ required further intervention (all of them were small procedures and were uneventfully done).

El-Nahas el al. (10) studied 39 patients submitted to PCNL who presented with bleeding requiring angiographic renal embolization and reported that solitary kidney, staghorn calculi, multiple tracts, and an inexperienced surgeon were significant risk factors for severe bleeding. In our study, a second tract was significantly related to bleeding. A more conservative approach avoiding more than one renal puncture in complex cases might be safer in these patients. Surgeon experience was not considered in our analysis, as all procedures in our institution were performed by a resident under supervision of an experienced staff urologist.

Jones et al. (11) in a study with 53 patients reported the safety of PCNL in solitary kidney patients showing a stone-free rate of $77.3 \%$ (defined by absence of residual calculi or fragments $\leq 2 \mathrm{~mm}$ ). The authors did not report any serious complications, except for one patient who had deterioration in renal function. More recently, Resorlu et al. (12) reported their experience with 16 patients with complex caliceal or staghorn stones in solitary kidney treated with PCNL. There were no significant intraoperative complications, but one patient had bleeding from an infundibular tear attributed to excessive kidney torquing. During the 1-year study period, no patients progressed to end-stage renal disease. They concluded that PCNL in solitary kidney with staghorn calculi is not only effective but also safe. In our study we had $3(11.1 \%)$ minor complications, two urinary 
fistulas and a double-J misplacement, which required further intervention. All of them were solved uneventfully.

PCNL in obese patients has its particularities that may be related to a harder patient's mobilization and positioning before surgery and a longer urinary tract length. These peculiarities could be linked to some difficulty to gain access to the collecting system and sometimes to a significant bleeding. In our study, BMI was not related to surgical complications or bleeding following PCNL in solitary kidneys. In fact, previous studies have shown that BMI does not impact on postoperative complications of PCNL $(13,14)$. Terrecialla-Ortiz et al. (14) in a prospective study including 225 PCNL reported no statistical significant difference in terms of complication or stone-free rate when patients were stratified according to their BMI. There were also no differences in failure to gain access, hospital stay, or need for auxiliary procedures. In this study, only operative time and radiation doses were higher in patients with increased BMI.

Canes et al. (15) studied the impact of PCNL on renal function and reported a series of 81 patients with solitary kidney submitted to 92 percutaneous procedures. In 64 (69.6\%) patients PCNL was done for stones, including staghorn calculi in 25 and renal or ureteral stones in 39 cases. In this study, percutaneous procedures were not associated with a worsening in renal function, which was similar to our findings. Wang et al. (16) reported their experience with PCNL in solitary kidney patients in prone (10 patients) and supine position (6 patients), showing that patient's position has no impact on surgical outcomes, which was again similar to our results.

In our study, the overall stone-free rate was $67 \%$. Although this number can be slightly lower compared to those reported by others authors $(12,15-18)$, the strict follow-up based on a NCCT to evaluate outcomes may explain that finding. Furthermore, it was similar to the results showed by CROES study in a global evaluation.

Recent published systematic review and meta-analysis showed that PCNL provides a higher stone free-rate when compared to RIRS. However, PCNL is also followed by a higher compli- cation rate and blood loss (19). In patients with solitary kidneys with kidney stones $>2.0 \mathrm{~cm}$, PCNL should be recommended as the first-line treatment option, although RIRS can be offered to selected patients who prefer to be submitted to staged procedures with low morbidity rate.

Our study has some limitations. It is a retrospective study with a limited number of patients. However, PCNL in solitary kidney is a relative rare procedure and prospective series are challenging to be done. Although it is not a large series, it has a reasonable number of patients and evidenced the association between multiple urinary tracts and bleeding, showing that a more conservative approach (single puncture procedure) might avoid blood transfusions. We did not compare our PCNL outcomes in solitary kidneys to those obtained from PCNL in patients with two functional kidneys; however, it would be complex to match these patients considering all variables which could be associated with intraoperative bleeding and postoperative complications.

\section{CONCLUSIONS}

PCNL in solitary kidneys is a challenging procedure, however a reasonable stone-free rate can be achieved with a low rate of significant complications. Multiple urinary tracts impacts on intraoperative bleeding and when there is the need for a second tract, a staged procedure is advised.

\section{ABBREVIATIONS}

$$
\begin{aligned}
& \text { ASA = American Society of Anesthesiology } \\
& \text { BMI = body mass index } \\
& \text { CROES = Clinical Research Office of the Endouro- } \\
& \text { logical Society } \\
& \text { eGFR = estimated Glomerular Filtration Rate } \\
& \text { MDRD = Modification of Diet in Renal Disease } \\
& \text { NCCT = noncontrast computed tomography } \\
& \text { PCNL = percutaneous nephrolithotomy } \\
& \text { RIRS = retrograde intrarenal surgery } \\
& \text { SWL = shock wave lithotripsy }
\end{aligned}
$$

\section{CONFLICT OF INTEREST}

None declared. 


\section{REFERENCES}

1. Scales CD Jr, Smith AC, Hanley JM, Saigal CS; Urologic Diseases in America Project. Prevalence of kidney stones in the United States. Eur Urol. 2012; 62:160-5.

2. Alexander RT, Hemmelgarn BR, Wiebe N, Bello A, Morgan C, Samuel S, et al. Kidney stones and kidney function loss: a cohort study. BMJ. 2012; 345:e5287.

3. el-Assmy A, el-Nahas AR, Hekal IA, Badran M, Youssef RF, Sheir KZ. Long-term effects of extracorporeal shock wave lithotripsy on renal function: our experience with 156 patients with solitary kidney. J Urol. 2008; 179:2229-32.

4. Atis G, Gurbuz C, Arikan O, Kilic M, Pelit S, Canakci C, et al. Retrograde intrarenal surgery for the treatment of renal stones in patients with a solitary kidney. Urology. 2013; 82:290-4

5. Bucuras V, Gopalakrishnam G, Wolf JS Jr, Sun Y, Bianchi G, Erdogru T, et al. CROES PCNL Study Group. The Clinical Research Office of the Endourological Society Percutaneous Nephrolithotomy Global Study: nephrolithotomy in 189 patients with solitary kidneys. J Endourol. 2012; 26:336-41.

6. Chandhoke PS, Albala DM, Clayman RV. Long-term comparison of renal function in patients with solitary kidneys and/or moderate renal insufficiency undergoing extracorporeal shock wave lithotripsy or percutaneous nephrolithotomy. J Urol. 1992; 147:1226-30.

7. Goel MC, Ahlawat R, Kumar M, Kapoor R. Chronic renal failure and nephrolithiasis in a solitary kidney: role of intervention. J Urol. 1997; 157:1574-7.

8. Basiri A, Shabaninia S, Mir A, Soltani MH. The safety and efficacy of percutaneous nephrolithotomy for management of large renal stones in single- versus double-functioning kidney patients. J Endourol. 2012; 26:235-8.

9. Thomas K, Smith NC, Hegarty N, Glass JM. The Guy's stone score--grading the complexity of percutaneous nephrolithotomy procedures. Urology. 2011; 78:277-81.

10. El-Nahas AR, Shokeir AA, El-Assmy AM, Mohsen T, Shoma AM, Eraky I, et al. Post-percutaneous nephrolithotomy extensive hemorrhage: a study of risk factors. J Urol. 2007; 177:576-9.
11. Jones DJ, Kellett MJ, Wickham JE. Percutaneous nephrolithotomy and the solitary kidney. J Urol. 1991; 145:477-9; discussion 479-80.

12. Resorlu B, Kara C, Oguz U, Bayindir M, Unsal A. Percutaneous nephrolithotomy for complex caliceal and staghorn stones in patients with solitary kidney. Urol Res. 2011; 39:171-6.

13. Tomaszewski JJ, Smaldone MC, Schuster T, Jackman SV, Averch TD. Outcomes of percutaneous nephrolithotomy stratified by body mass index. J Endourol. 2010; 24:547-50.

14. Torrecilla Ortiz C, Meza Martínez Al, Vicens Morton AJ, Vila Reyes $\mathrm{H}$, Colom Feixas S, Suarez Novo JF, et al. Obesity in percutaneous nephrolithotomy. Is body mass index really important? Urology. 2014; 84:538-43.

15. Canes D, Hegarty NJ, Kamoi K, Haber GP, Berger A, Aron M, et al. Functional outcomes following percutaneous surgery in the solitary kidney. J Urol. 2009; 181:154-60.

16. Wang $Y$, Hou $Y$, Jiang F, Wang $Y$, Wang C. Percutaneous nephrolithotomy for staghorn stones in patients with solitary kidney in prone position or in completely supine position: a single-center experience. Int Braz J Urol. 2012; 38:788-94.

17. Wong KA, Sahai A, Patel A, Thomas K, Bultitude M, Glass J. Is percutaneous nephrolithotomy in solitary kidneys safe? Urology. 2013; 82:1013-6.

18. Akman T, Binbay M, Tekinarslan E, Ozkuvanci U, Kezer C, Erbin A, et al. Outcomes of percutaneous nephrolithotomy in patients with solitary kidneys: a single-center experience. Urology. 2011; 78:272-6.

19. De S, Autorino R, Kim FJ, Zargar H, Laydner H, Balsamo $\mathrm{R}$, et al. Percutaneous nephrolithotomy versus retrograde intrarenal surgery: a systematic review and meta-analysis. Eur Urol. 2015; 67:125-37.
Correspondence address: Fábio César Miranda Torricelli, MD Av. Vereador Jose Diniz, 3300 / 208 Sao Paulo, SP, 04604-006, Brazil

Telephone: +55 11 5533-4900

E-mail: fabio_torri@yahoo.com.br 